\section{Cureus}

Received 05/20/2019

Review began 05/23/2019

Review ended 06/03/2019

Published 06/21/2019

\section{(c) Copyright 2019}

Diaz Casas et al. This is an open access article distributed under the terms of the Creative Commons Attribution License CC-BY 3.0., which permits unrestricted use, distribution, and reproduction in any medium, provided the original author and source are credited.

\title{
Clinical Behavior of Triple Negative Breast Cancer in a Cohort of Latin American Women
}

Sandra Diaz Casas ${ }^{1}$, Eder Lancheros García ${ }^{1}$, Andrés Sanchéz Campo ${ }^{2}$, Ricardo Sanchez Pedraza $^{3}$, Vivian Roman Vasquez ${ }^{4}$, Sara D. Mendoza ${ }^{5}$, Javier Angel Aristizabal ${ }^{1}$, Carlos Lehmann Mosquera ${ }^{1}$, Carlos Duarte Torres ${ }^{1}$, Juan C. Vergel ${ }^{1}$

1. Breast and Soft Tissue Clinic, Instituto Nacional de Cancerologia, Bogotá D.C., COL 2. Breast and Soft Tissue Clinic, Clinica De Occidente, Bogotá D.C., COL 3. Office of the Deputy Director for Research, Epidemiological Surveillance, Promotion and Prevention, Instituto Nacional de Cancerologia, Bogotá D.C., COL 4. Breast Surgery, Fundacion Valle Del Lili, Bogotá D.C., COL 5. Oncology, Instituto Nacional de Cancerologia, Bogotá D.C., COL

$\square$ Corresponding author: Sara D. Mendoza, mendozadiaz.sara@gmail.com Disclosures can be found in Additional Information at the end of the article

\section{Abstract}

Introduction: Breast cancer is a worldwide public health problem. In Colombia, there are 13,000 new cases, having the highest incidence and mortality among cancers. This article describes the clinical behavior of patients with triple negative breast cancer (TNBC) treated at the National Cancer Institute (NCI) in Bogota, Colombia.

Methods: A historical cohort and analytical study that included elderly patients diagnosed with TNBC treated at the National Cancer Institute Functional Breast Cancer Unit (NCI-FBCU) was conducted.

Results: Of the 1,066 patients registered in the unit from September 1st 2013 to December 31st 2016: 146 (13.7 \%) had triple negative tumors. The average age was 57.3 years; $61 \%$ of patients had locally advanced tumors. The majority of patients received neoadjuvant chemotherapy as their first treatment (69.1\%), and in $41.2 \%$ of the cases platinum was added to the chemotherapy regimen. The most common surgery conducted was modified radical mastectomy in $57.8 \%$ of cases. The pathological complete response (pCR) (Chevallier 1 and 2) was reached in $22.6 \%$ and, in this group of patients, a greater overall survival (OS) was found [hazard ratio (HR) $0.08,95 \%$ CI $0.01-0.63 ; p=0.016]$. Progression of the disease occurred in $36.5 \%$ of cases, being lungs the most frequent location (44.4\%). The death incidence rate was 1.21 deaths per 100 patients/month. The median event-free survival (EFS) was 18.2 months.

Conclusion: TNBC occurs in Latin American women at advanced clinical stages with aggressive clinical behavior, with lower OS rates, and higher risk of metastasis compared to other molecular subtypes.

Categories: Oncology

Keywords: breast neoplasm, cohort, survival, chemotherapy, surgery

\section{Introduction}

Breast cancer is a worldwide public health problem with 2.1 million new cases diagnosed in

How to cite this article

Diaz Casas S, Lancheros García E, Sanchéz Campo A, et al. (June 21, 2019) Clinical Behavior of Triple 
2018, according to data from the latest GLOBOCAN update. It ranks first in incidence of female cancer worldwide with $24.2 \%$, and is the leading cause of death from cancer in women (15\%) [1].

In Colombia, the number of new cases of breast cancer in 2017 accounted for $13.1 \%$ of all cancers in both sexes; being the most common cancer in our country with 13,380 women diagnosed per year. It also ranks first in mortality accounting for $15.9 \%$ of all cases [1].

The National Cancer Institute (NCI) is the most important reference cancer center in Colombia. At the National Cancer Institute Functional Breast Cancer Unit (NCI-FBCU), patients who were admitted to the NCI for the first time with a diagnosis of cancer and who have not yet received any type of previous treatment, are conducted through diagnostic services of the hospital and then introduced at a meeting where a Breast Surgeon and a Clinical Oncologist participate in order to define the patients' treatments; in addition, the coordinator of NCI-FBCU (a Nurse Oncologist) is responsible for ensuring management, monitoring, and completion of the database where treatments, dates, and outcomes are recorded.

From September 1st 2013 to December 31st 2016, 1,066 new cases of breast cancer were admitted to the functional unit, of which 146 were triple negative tumors (TNBC), accounting for $13.7 \%$ of all cases diagnosed in that period of time.

Current scientific evidence establishes that TNBC accounts for $15 \%$ of all cancers diagnosed and affects mainly women who are Hispanic, Afro-American, pre-menopausal, or carriers of BRCA gene mutations [2].

A retrospective cohort study conducted in 2008 at the MD Anderson Cancer Center, included 491 patients with breast cancer and documented a mutation in the BRCA gene in $17.5 \%$ of the cases; of these, $57.6 \%$ were TNBC, the most frequent mutation in this subgroup of patients was in the BRCA1 gene with $57.1 \%$ of cases, in the BRCA2 with $23.3 \%$ of the cases, and $13.8 \%$ were nonmutated [3].

Triple negative breast cancer is a tumor that expresses less than $1 \%$ estrogens and progestogens, and is HER2-negative; it is a subtype with greater aggressiveness in its appearance, associated with greater tumor size, high histological grade and frequently with lymph node involvement [2], because of this it has a high risk of distant metastasis, especially to lungs, brain, and liver. The risk of recurrence in the first three years after starting treatment is greater compared to other molecular subtypes of breast cancer [4-5].

Triple negative breast cancer is a heterogeneous disease by itself. In 2011, Lehmann et al. identified six specific subtypes by using gene expression profiles through microarray technology. These subtypes are: basal-like 1 (BL1), basal-like 2 (BL2), immunomodulatory (IM), mesenchymal (M), mesenchymal stem cell (MSL), and luminal androgen receptor (LAR) [6].

Researchers concluded that each subtype described different clinical behavior, sensitivity to chemotherapy, and overall survival (OS) [6].

Parallel to this classification, Burstein et al. developed a DNA and RNA sequence in 200 tumors with TNBC, by which they identified four subtypes: luminal androgens receptor, mesenchymal, basal immunosuppressant, and basal immunoactive [7].

In this classification, the basal-like immunosuppressant subtype has the worst prognosis, and the basal-like immunoactive has the best prognosis, in terms of OS and event-free survival (EFS) [7]. 
The purpose of this article is to describe the clinical behavior of TNBC patients who are treated at the NCI-FBCU during the period described above.

\section{Materials And Methods}

An observational, analytical, historical, and cohort study was conducted. The cohort was defined as "patients with a confirmed diagnosis of TNBC, who were admitted into NCI-FCBU in Bogota, Colombia, and received their entire treatment during a period of three years (September 1st 2013 until December 31st 2016).” Patients with luminal breast cancer, HER2positive, and those who did not receive full treatment within the NCI-FCBU, were excluded. This work was approved by the NCI Institutional Ethics Committee.

Database from the NCI-FBCU and records from the NCI digital clinical history (SAP ${ }^{\circledR}$, SAP SE, Walldorf, Germany), were used as a source of information for the selection of patients. Two coresearchers, specialists in breast surgery, extracted data. We reviewed treatment records, start date and follow-up, as well as one-by-one records of each SAP ${ }^{\circledR}$ patient's clinical history, on the following aspects: registered demographic data, pathology report of the biopsy and surgical specimen, chemotherapy records, radiotherapy records, follow-up consultation from the Breast and Soft Tissue Clinic, Clinical Oncology, Radiotherapy, Palliative Care, and Emergency.

The clinical response was assessed with the scale of the World Health Organization (WHO) [8], and the pathological response with Chevallier criteria [9].

The variables included in this study were: age, health assurance system, first-degree family history with breast cancer, body mass index, menopausal status, pathological characteristics of the biopsy, clinical stage of the disease, initial treatment received, clinical response obtained according to the WHO scale, neoadjuvant chemotherapy regimen administered, surgery performed, pathological response according to Chevallier criteria, use of adjuvant radiotherapy, localization an treatment of recurrence, and follow-up [EFS, progression-free survival (PFS) and OS].

Using absolute and relative frequency measures, a descriptive analysis of the categorical and nominal variables was carried out. With regard to continuous variables, measures of central tendency and measures of dispersion were used.

The clinical behavior of patients with TNBC was assessed, and the proportion of patients who received cancer treatment, their response to neoadjuvant chemotherapy, and percentage of their pCR were calculated. The connection between categorical variables was assessed by using Fisher's exact tests.

Survival analyses for the corresponding events were quantified: death from any cause, eventfree period, and progression-free period. For EFS and OS, the date of surgical procedure was taken as the time of admission to the cohort. In the case of PFS, the date of the latest cycle of primary chemotherapy was taken as the time of admission to the cohort. Events were taken as numerators to estimate incidence density rates, taking as denominator the total time contributed by patients of the cohort.

These rates were estimated taking into account the risk of bias due to the presence of differential follow-up. Incidence estimators were reported with $95 \%$ confidence intervals. Survival functions were estimated for each of the three outcomes, by using the Kaplan-Meier method. These functions took into account the loss of follow-up, handled as censors on the right. 


\section{Cureus}

In addition, log-rank test was used to compare survival functions, and significance levels of 5\% were used for all hypothesis tests.

Statistical analysis of data was performed using the Stata Statistical Software: Release 12 (StataCorp 2011, StataCorp LP, College Station, TX), licensed for the NCI.

\section{Results}

Of the 1,066 patients registered in the NCI-FBCU from September 2013 to December 2016, 146 (13.7\%) had TNBC; 23 patients (15.7\%) were excluded due to the following reasons: nine had received some type of treatment outside the NCI; eight died before starting treatment; three patients had tumors with overexpression of HER2; two patients rejected the treatment; and one received incomplete treatment. This analysis included 123 patients: most of them belong to the subsidized health system $(59.3 \%, \mathrm{n}=73)$; their average age was $57.3(22-88)$ years old; the majority was postmenopausal $(73.2 \%, \mathrm{n}=90)$; a genetic study was ordered to 45 patients (36.5\%) and it was conducted in 32 of them; most had complete gene sequencing $(59.4 \%, \mathrm{n}=$ 19). Regarding clinical stage, $61.0 \%(n=75)$ of cases were locally advanced tumors, being stage IIIB $(35.0 \%, n=43)$ the most frequent. $18.7 \%(n=23)$ of the patients in this series of cases were admitted with metastatic disease (Table 1).

\section{Characteristics}

Type of health assurance system

Subsidized

Contributive

Age (years)
$\%$

40.7 50

Antecedent of breast cancer on first degree family

No

Yes

9.8

10.6

No Data

Overweight (25.1-29.9)

Class I obesity (30-34.9)

Class II obesity (35-39.9)

Morbid obesity (>40)

Genetic study

Requested test

Performed test

BRCA 1/2 complete sequencing 


\section{Cureus}

Colombia profile

Others

Histological type

NOS ductal

Pure ductal

Metaplastic

Medullar

Papillary

Others

Differentiation grade

I

II

III

Lymphovascular invasion

Absent

Androgen receptor report

Not reported

Negative

CK report (5/6)

Not reported

Negative

Positive

Ki 67 (\%)

$>60$

$41-60$

$21-40$

$<20$

No data

Tumor size (T)
77.2

99.2

0.8

67.5

15.4

34.4 11

.2 2

$0.8-1$

$18.7 \quad 23$

99

3

3.3

3.3

4

$10.6 \quad 13$

$4.1 \quad 5$

$2.4 \quad 3$ 


\section{Cureus}

$\mathrm{T} 1$

T2

T3

$\mathrm{T} 4 \mathrm{~b}$

$\mathrm{T} 4 \mathrm{c}$

T4d

Lymph node involvement (N)

No

N1

N2a

N2b

N3a

N3b

N3c

Metastatic involvement

M0

M1

Location of metastasis

Multiple locations

Lungs

Bones

Skin

Nonregional lymph nodes

Liver

CNS

Others

Clinical stage

I

IIA

IIB

IIIA
4.1

26.8

$13.0 \quad 16$

52.8

1.62

1.6

2

26.0

32

$26.8 \quad 33$

$35.8 \quad 44$

$0.0 \quad 0$

$1.6 \quad 2$

2.43

7.3

9

$81.3 \quad 100$

$18.7 \quad 23$

$47.8 \quad 11$

$47.8 \quad 11$

$34.8 \quad 8$

$34.8 \quad 8$

$21.5 \quad 5$

$17.4 \quad 4$

$4.3 \quad 1$

12.93

$4.1 \quad 5$

$16.3 \quad 20$

$\begin{array}{ll}7.3 & 9\end{array}$

$13.8 \quad 17$ 


\section{Cureus}

IIIB

IIIC

IV

\section{TABLE 1: Characteristics of patients diagnosed with TNBC at the NCI-FBCU.}

TNBC, Triple negative breast cancer; NCI-FBCU, National Cancer Institute Functional Breast Cancer Unit.

The majority of patients received chemotherapy as initial treatment $(87.0 \%, \mathrm{n}=107) ; 69.1 \%(\mathrm{n}=$ $85)$ had neoadjuvant intention and $17.9 \%(n=22)$ had primary intention, because of the advanced clinical status of most patients. The most used regimen was anthracyclines, cyclophosphamide, and taxanes (AC-T) in 41.2\% $(n=35)$ of patients; and in $41.1 \%$ of cases $(n=$ 35) carboplatin was added to the chemotherapy regimen. The most performed surgical procedure was modified radical mastectomy $(57.8 \%, \mathrm{n}=59)$ of patients. pCR was reported in $22.6 \%(n=23)$ of patients. The group of patients in which carboplatin chemotherapy regimens were included, pCR was found in 11 of the 35 patients (31.4\%), without statistical significance $(p=0.13)$. Of the nine patients with mutation in the BRCA1 and BRCA2 genes, seven had neoadjuvant chemotherapy, and of these, only four received regimens that included platinum, and in addition, only one of these four patients had a pCR. A better OS was found in patients who presented pCR compared to patients who presented residual infiltrative disease in the breast and/or axillary metastatic lymph node involvement in the surgical pathology (Chevallier 3-4) (HR 0.08, 95\% CI 0.01-0.63; $\mathrm{p}=0.016$ ). The residual tumoral size in the surgical pathology ranged between 0 and $20 \mathrm{~cm} ; 60.8 \%(n=62)$ of patients who were taken into surgery had negative node involvement in the surgical piece.

Regarding the adjuvant, $57.8 \%(\mathrm{n}=71)$ of patients in this cohort received radiotherapy; $17.9 \%$ ( $\mathrm{n}$ $=22$ ) received adjuvant chemotherapy. $9.8 \%(n=12)$ of patients of the two previous groups received both treatments sequentially (Table 2 ).

Types of treatment

First treatment received

Neoadjuvant chemotherapy

Primary chemotherapy

Radical surgery

Conservative surgery

Radiotherapy

$0.8 \quad 1$

Neoadjuvant chemotherapy regimen administered

Adriamycin-Cyclophosphamide-Taxane (AC-T) 


\section{Cureus}

Adriamycin-Cyclophosphamide (AC)

$2.4 \quad 2$

Others

Primary chemotherapy regimen administered

Taxane-Carboplatin (TC)

45.510

Adriamycin-Cyclophosphamide-Taxane (AC-T)

18.24

Adriamycin-Cyclophosphamide-Taxane-Carboplatin (AC-TC)

$13.6 \quad 3$

Adriamycin-Cyclophosphamide (AC)

$9.1 \quad 2$

Others

Type of surgical treatment

Modified radical mastectomy

$57.8 \quad 59$

Quadrantectomy + axillary dissection

Quadrantectomy + sentinel lymph node

Simple mastectomy + sentinel lymph node

$7.8 \quad 8$

Radical mastectomy

$2.0 \quad 2$

Breast reconstruction

Not performed

$84.6 \quad 104$

Performed

$15.4 \quad 19$

TRAM

$52.6 \quad 10$

Expander/prosthesis

26.35

Latissimus dorsi

15.83

Latissimus dorsi + prosthesis

$5.1 \quad 1$

Pathological response to neoadjuvant chemotherapy (Chevallier criteria)

1

20.6

2

$2.0 \quad 2$

3

32.433

4

$24.5 \quad 25$

Not applicable

20.6

Number of lymph nodes reported in the surgical piece of the axilla 


\section{Cureus}

$>10$ nodes

Type of adjuvant treatment received

Exclusive radiotherapy

Chemotherapy and sequential radiotherapy

Exclusive chemotherapy

Adjuvant radiotherapy

50 Gy

$>50$ Gy

$<50$ Gy

Adjuvant chemotherapy

Adriamycin-Cyclophosphamide (AC)

Taxane-Carboplatin (TC)

Capecitabine

Taxane

\section{TABLE 2: Types of treatments administered to patients diagnosed with TNBC at the} NCI-FBCU.

In this cohort, $36.6 \%(n=45)$ presented disease progression mostly with distant metastasis (77.7\%, $n=35)$, being lungs the most frequent location $(44.4 \%, n=20)$; and $42.2 \%(n=18)$ of patients had more than two metastatic locations. Local recurrence occurred more frequently in patients taken into radical surgery $(13 / 15 ; \mathrm{p}=0.35)$, which was not statistically significant. $11.1 \%(n=5)$ of patients did not receive any type of treatment for the management of recurrence. $87.1 \%(n=34)$ of patients in whom the disease progressed, received some type of treatment; chemotherapy was administered to $25.6 \%(n=10)$; radiotherapy to $38.5 \%(n=15)$; and surgical management of local and/or regional recurrence was performed in $18.0 \%(n=7)$ of cases (Table 3). 


\section{Cureus}

\section{Location of recurrence}

Local

Regional

Distance

Specific organ involvement

Lungs

Bones

Central nerve system

Nonregional lymph nodes

Soft tissue

Liver

Skin

Pleura

Others

TABLE 3: Location of recurrence within patients diagnosed with TNBC at the NCIFBCU.

At 24-months follow-up: $48.0 \%(\mathrm{n}=59)$ of patients were alive without active disease; $12.2 \%(\mathrm{n}$ $=15)$ were alive with disease; $28.5 \%(n=35)$ died as a consequence of the disease; $6.5 \%(n=8)$ died due to other causes; and $4.9 \%(n=6)$ of patients were lost to follow-up (failure to attend controls). $60.0 \%(\mathrm{n}=27)$ of patients with progression of the disease (local, regional, or systemic) died during the time of study.

Overall survival for the group of patients with early and locally advanced disease, contributed a total of 2,384 months of follow-up, time during which 29 deaths occurred.

The time at risk had a median of 22.3 months (0.7-56.7 months). The incidence death rate was of 1.21 deaths per 100 patients / month (95\% CI 0.84-1.8) (Figure 1). 


\section{Cureus}

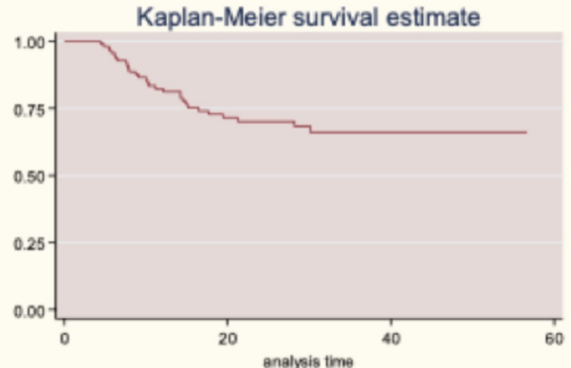

Overall survival

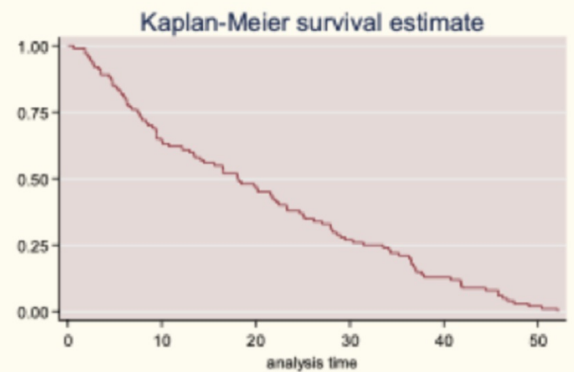

Disease-free survival

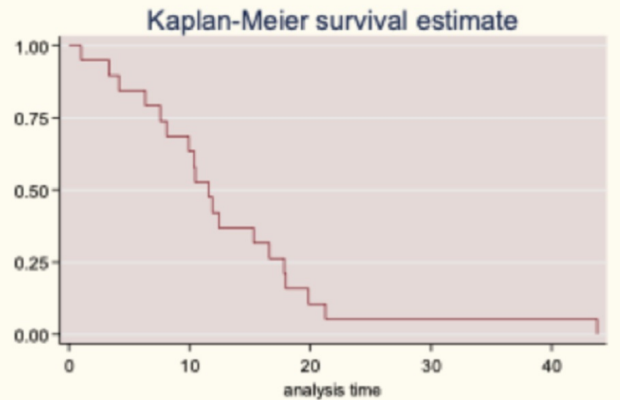

Progression-free survival

\section{FIGURE 1: Overall survival, disease-free survival, and progression-free survival within the cohort of patients diagnosed with TNBC at the NCI-FBCU.}

The analysis of EFS was performed in patients with early and locally advanced diseases, thus excluding from the analysis patients with metastatic disease; this group of patients contributed 2,051 months of follow-up, time during which 100 relapses occurred. The median EFS was 18.2 months. The time at risk had a median of 18.1 months (0.7-52 months). The incidence death rate was of 4.88 deaths per 100 patients / month (95\% CI 4-5.9) (Figure 1).

The analysis of PFS in stage IV patients included 21 patients (two were excluded because stage IV was by skin and chemotherapy was ordered with a neoadjuvant intention). Patients contributed 253.2 months, time during which 19 progressions occurred. The median PFS was 11.6 months. The time at risk had a median of 10.5 months (0.4-43.8 months). Progression incidence rate was of 7.5 progressions per 100 patients / month (95\% CI 4.8-11.8) (Figure 1).

There is no significant difference in OS functions and in EFS with respect to age over or under 50 years. A higher mortality rate was found as the clinical stage increased. The difference in survival functions is significant (Log-rank test: $\chi 2=14.14(2) ; p=0.0009)$ (Figure 2). A higher recurrence rate was found as the clinical stage increased. The difference in OS functions is significant (Log-rank test: $\chi 2=25.77$ (2); $p=0.0009$ ). As well, patients diagnosed with stage IV have a higher risk of death compared to patients with early and locally advanced tumors (HR 3.4, 95\% CI 1.3-9-0; $\mathrm{p}=0.013$ ) (Figure 2). 


\section{Overall Survival}

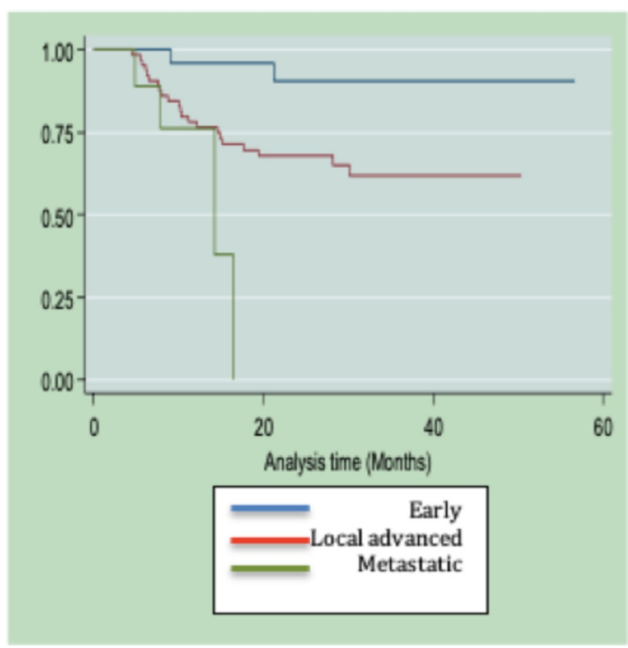

\section{Disease-free survival}

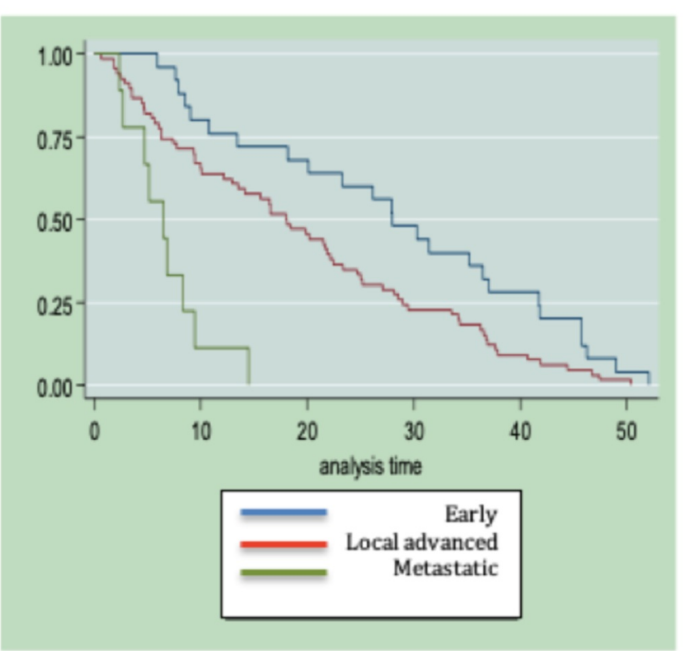

FIGURE 2: Overall survival and disease-free survival in relation to clinical stage within the cohort of patients diagnosed with TNBC at the NCI-FBCU.

\section{Discussion}

Triple negative breast cancer is an entity that has become a therapeutic challenge for cancer centers and accounts for $15 \%$ of all breast tumors. It registers a more aggressive biological behavior, higher mortality rates, and risk of metastasis within the first three years after diagnosis [2].

The NCI is a reference cancer center at national level, and as Colombia is a developing country, most patients enter with locally advanced stages of the disease. For this cohort, specifically, $61.0 \%(n=75)$ of cases were locally advanced tumors, and $18.7 \%(n=23)$ were stage IV.

Unfortunately, the Institution's diagnostic protocol includes only four immunohistochemistry markers, and it is difficult from the administrative point of view that healthcare providers authorize additional markers, reason why only $7 \%$ of our patients have other IHC markers, and it is a limitation of our study to classify these tumors according to the classification described by Lehmann et al., as we do not have enough resources to do so [6].

Case series worldwide show that unlike luminal tumors where bone is the most common location of metastatic disease [10], in TNBC patients the most common sites for metastasis are lungs and brain [11-12]; which is very similar to that observed in this work, where lungs were the most common location of appearance of the disease at stage IV (47.8\%) as well as for recurrence (44.4\%).

Pathological complete response to neoadjuvant chemotherapy in this cohort is much lower (22.6\%) than that registered in randomized clinical trials and meta-analyses ranging from 33\% to $42 \%$ [13-16], which could be attributed to advanced stages of the disease, and in some cases due to problems with the authorization of medicines by the insurer. In this cohort, the pCR when using platinum regimens was of $31.5 \%$, a very low value compared to results presented in the GeparSixto and CALGB 40606 [17-18]. 
In the German group's study, GeparSixto administered neoadjuvant chemotherapy with paclitaxel, liposomal doxorubicin and bevacizumab, with or without weekly carboplatin, in 315 patients with TNBC, finding $\mathrm{pCR}$ in $53.2 \%$ of patients treated with carboplatin, compared to $36.9 \%$ of those who did not receive the medication $(p=0.005)$; reporting statistically significant differences in the two arms of the study, concluding that the addition of a carboplatin to neoadjuvant therapy with a taxane and/or anthracycline, significantly increases the rate of pCR in this group of patients [17].

Likewise, CALGB 40603 assessed weekly paclitaxel with or without carboplatin and/or bevacizumab followed by a dose of doxorubicin and cyclophosphamide, thus reporting an increase in pCR rate from $46 \%$ to $60 \%$ in favor of the study branch that received carboplatin ( $\mathrm{p}=$ 0.018). In this study, pCR did not impact OS [18].

In the meta-analysis by Cortazar et al. [13], OS is reported in the subgroup of patients with TNBC with an HR: 0.16 (0.11-0.25) and EFS with an HR: 0.24 (0.18-0-33). In our study, OS was of $92 \%$ in patients who achieved pCR.

In this cohort, radical surgery was performed in a higher percentage, which is explained by the higher frequency of locally advanced tumors. In this same sense, the low percentage of immediate breast reconstruction draws attention, which is attributed mainly to administrative problems with health providers, availability of the plastic surgeon and little interest on the part of patients to carry out their immediate reconstructive procedure. Moreover, local recurrence was higher in patients taken to radical surgery, which is also explained by the advanced stage of their disease.

In our cohort, only two patients received adjuvant treatments with capecitabine, given that we still did not have results of the CREATE X study, which was published in June of 2017.

Masuda et al. reported in the subgroup of patients diagnosed with TNBC (n: 286) with residual disease, that the administration of six cycles of capecitabine as adjuvant treatment, positively impacted five-year event-free survival (69.8\% vs. $56.1 \%$ ) (HR $0.58,95 \%$ CI $0.39-0.87$ ), and $78.8 \%$ vs. $70.3 \%$ OS (HR 0.52 , 95\% CI 0.30-0.87) [19].

In our cohort, $28.5 \%$ of patients died due to the disease, and $12.2 \%$ are in active treatment for their metastatic disease. Lin et al. evaluated 12,902 women by comparing patients with positive-hormone-receptor tumors and patients with TNBC, reporting a worse OS in patients with TNBC, in a study published in 2012 (HR 2.72, 95\% CI 2.39-3.10), and an increased risk of death in the first two years after diagnosis (HR 6.10, CI 95\% 4.81-7.74) [20].

However, this risk decreases with time, with a 10 -year relapse-free interval of $97 \%$ and a 15year interval of $95 \%$. Regarding relapse-free survival, it was of $91 \%$ and $83 \%$, respectively [ $20-$ 21].

Overall survival of TNBC is lower compared to other molecular subtypes. In luminal A tumors is of $92.5 \%$, in luminal B HER2-positive is $90.3 \%$, in pure HER2 (HER2-positive, negativehormone-receptor) $82.7 \%$, and in TNBC is of $77 \%$ [22]. In our cohort, disease progression appeared in $36.6 \%$ of cases, and mortality was higher in locally advanced and metastatic tumors. No connection was found with age, previous data that coincide with the work of Li et al. [23].

Breast cancer research is currently aimed at patients with TNBC, especially in the field of immunotherapy both in metastatic disease and in the neoadjuvant and adjuvant setting [2426]. 
Among limitations of this work, we can find derivatives of the retrospective studies to establish causal relationships, and the difficulty of conducting follow-up on patients due to changes in contracting with other institutions. Therefore, the size of the sample would not allow subsequent statistical analyzes with multiple covariates and their association with the outcome.

\section{Conclusions}

Triple negative breast cancer is a heterogeneous and aggressive disease with a worse OS rate and a higher risk of metastasis compared with other molecular subtypes, findings that coincide with the results of our cohort.

\section{Additional Information Disclosures}

Human subjects: Consent was obtained by all participants in this study. Institutional Ethics Committee of the National Cancer Institute of Bogota, Colombia. issued approval 002 2018. We did not receive any payment from a third party for any aspect of the submitted work. We do not have any financial relationships with entities. We do not have any patents relevant to the work. This work was approved by the Institutional Ethics Committee of the National Cancer Institute of Bogota, Colombia. . Animal subjects: All authors have confirmed that this study did not involve animal subjects or tissue. Conflicts of interest: In compliance with the ICMJE uniform disclosure form, all authors declare the following: Payment/services info: All authors have declared that no financial support was received from any organization for the submitted work. Financial relationships: All authors have declared that they have no financial relationships at present or within the previous three years with any organizations that might have an interest in the submitted work. Other relationships: All authors have declared that there are no other relationships or activities that could appear to have influenced the submitted work.

\section{Acknowledgements}

To chief Martha Orozco, Oncologist Nurse at the National Cancer Institute Functional Breast Cancer Unit, for her great contribution to development of the database. To Maribel Charris for follow-up and phone calls to cohort patients. To Dominga Ariza, NCI research monitor.

\section{References}

1. Bray F, Ferlay J, Soerjomataram I, et al.: Global cancer statistics 2018: GLOBOCAN estimates of incidence and mortality worldwide for 36 cancers in 185 countries. CA Cancer J Clin. 2018, 10.3322/caac. 21492

2. Moore-Smith L, Forero-Torres A, Stringer-Reasor E: Future developments in neoadjuvant therapy for triple-negative breast cancer. Surg Clin North Am. 2018, 98:773-785. https://www.ncbi.nlm.nih.gov/pubmed/30005773

3. Atchley DP, Albarracin CT, Lopez A, et al.: Clinical and pathologic characteristics of patients with BRCA-positive and BRCA-negative breast cancer. J Clin Oncol. 2008, 26:4282-4288. 10.1200/JCO.2008.16.6231

4. Dent R, Trudeau M, Pritchard KI, et al.: Triple-negative breast cancer: clinical features and patterns of recurrence. Clin Cancer Res. 2007, 13:4429-4434. 10.1158/1078-0432.CCR-06-3045

5. Ahn SG, Kim SJ, Kim C, Jeong J: Molecular classification of triple-negative breast Cancer. J Breast Cancer. 2016, 19:223-230. 10.4048/jbc.2016.19.3.223

6. Lehmann BD, Bauer JA, Chen X, et al.: Identification of human triple-negative breast cancer subtypes and preclinical models for selection of targeted therapies. J Clin Invest. 2011, 121:2750-2767. 10.1172/JCI45014

7. Burstein MD, Tsimelzon A, Poage GM, et al.: Comprehensive genomic analysis identifies novel subtypes and targets of triple-negative breast cancer. Clin Cancer Res. 2015, 21:1688-1698. 


\subsection{8/1078-0432.CCR-14-0432}

8. Miller AB: Breast cancer. Cancer. 1981, 47:1109-1113.

9. Chevallier B, Roche H, Olivier JP, et al.: Inflammatory breast cancer. Pilot study of intensive induction chemotherapy (FEC-HD) results in a high histologic response rate. Am J Clin Oncol. 1993, 16:223-228.

10. Kennecke H, Yerushalmi R, Woods R, et al.: Metastatic behavior of breast cancer subtypes . J Clin Oncol. 2010, 28:3271-3277. 10.1200/JCO.2009.25.9820

11. Smid M, Wang Y, Zhang Y, et al.: Subtypes of breast cancer show preferential site of relapse . Cancer Res. 2008, 68:3108-3114. 10.1158/0008-5472.CAN-07-5644

12. Dent R, Hanna WM, Trudeau M, et al.: Pattern of metastatic spread in triple-negative breast cancer. Breast Cancer Res Treat. 2009, 115:423-428. 10.1007/s10549-008-0086-2

13. Cortazar P, Zhang L, Untch M, et al.: Pathological complete response and long-term clinical benefit in breast cancer: the CTNeoBC pooled analysis. Lancet. 2014, 384:164-172.

10.1016/S0140-6736(13)62422-8

14. Omarini C, Guaitoli G, Pipitone S, et al.: Neoadjuvant treatments in triple-negative breast cancer patients: where we are now and where we are going. Cancer Manag Res. 2018, 10:91103. 10.2147/CMAR.S146658

15. Liedtke C, Mazouni C, Hess KR, et al.: Response to neoadjuvant therapy and long-term survival in patients with triple-negative breast cancer. J Clin Oncol. 2008, 26:1275-1281. 10.1200/JCO.2007.14.4147

16. Huober J, von Minckwitz G, Denkert C, et al.: Effect of neoadjuvant anthracycline-taxanebased chemotherapy in different biological breast cancer phenotypes: overall results from the GeparTrio study. Breast Cancer Res Treat. 2010, 124:133-140. 10.1007/s10549-010-1103-9

17. von Minckwitz G, Schneeweiss A, Loibl S, et al.: Neoadjuvant carboplatin in patients with triple-negative and HER2-positive early breast cancer (GeparSixto; GBG 66): a randomised phase 2 trial. Lancet Oncol. 2014, 15:747-756. 10.1016/S1470-2045(14)70160-3

18. Sikov WM, Berry DA, Perou CM, et al.: Impact of the addition of carboplatin and/or bevacizumab to neoadjuvant once-per-week paclitaxel followed by dose-dense doxorubicin and cyclophosphamide on pathologic complete response rates in stage II to III triple-negative breast cancer: CALGB 40603 (Alliance). J Clin Oncol. 2015, 33:13-21. 10.1200/JCO.2014.57.0572

19. Masuda N, Lee SJ, Ohtani S, et al.: Adjuvant capecitabine for breast cancer after preoperative chemotherapy. N Engl J Med. 2017, 376:2147-2159. 10.1056/NEJMoa1612645

20. Lin NU, Vanderplas A, Hughes ME, et al.: Clinicopathologic features, patterns of recurrence, and survival among women with triple-negative breast cancer in the National Comprehensive Cancer Network. Cancer. 2012, 118:5463-5472. 10.1002/cncr.27581

21. Reddy SM, Barcenas CH, Sinha AK, et al.: Long-term survival outcomes of triple-receptor negative breast cancer survivors who are disease free at 5 years and relationship with low hormone receptor positivity. Br J Cancer. 2018, 118:17-23. 10.1038/bjc.2017.379

22. Howlader N, Cronin KA, Kurian AW, et al.: Differences in breast cancer survival by molecular subtypes in the United States. Cancer Epidemiol Biomarkers Prev. 2018, 27:619-626. 10.1158/1055-9965.EPI-17-0627

23. Li X, Yang J, Peng L, et al.: Triple-negative breast cancer has worse overall survival and causespecific survival than non-triple-negative breast cancer. Breast Cancer Res Treat. 2017, 161:279-287. 10.1007/s10549-016-4059-6

24. De La Cruz LM, Czerniecki BJ: Immunotherapy for breast cancer is finally at the doorstep: immunotherapy in breast cancer. Ann Surg Oncol. 2018, 25:2852-2857. 10.1245/s10434-0186620-5

25. Nanda R, Chow LQ, Dees EC, et al.: Pembrolizumab in patients with advanced triple-negative breast cancer: phase Ib KEYNOTE-012 study. J Clin Oncol. 2016, 34:2460-2467. 10.1200/JCO.2015.64.8931

26. Schmid P, Adams S, Rugo HS, et al.: Atezolizumab and nab-paclitaxel in advanced triplenegative breast cancer. N Engl J Med. 2018, 379:2108-2121. 10.1056/NEJMoa1809615 